\title{
Effects of Temporal Modeling on the Statistical Uncertainty of Spatiotemporal Distributions Estimated Directly from Dynamic SPECT Projections
}

\author{
Bryan W. Reutter ${ }^{\dagger}$, Grant T. Gullberg ${ }^{\ddagger}$, and Ronald H. Huesman ${ }^{\dagger}$ \\ ${ }^{\dagger}$ Center for Functional Imaging, Lawrence Berkeley National Laboratory \\ University of California, Berkeley, CA 94720, USA \\ ${ }_{\ddagger}^{\ddagger}$ Medical Imaging Research Laboratory, Department of Radiology \\ University of Utah, Salt Lake City, UT 84108, USA
}

\section{INTRODUCTION}

$\mathrm{A}$ RTIFACTS can result when reconstructing a dynamic image sequence from inconsistent single photon emission computed tomography (SPECT) projections acquired by a slowly rotating gantry. The artifacts can lead to biases in kinetic parameters estimated from time-activity curves generated by overlaying volumes of interest on the images. To overcome these biases in conventional image based dynamic data analysis, we have been investigating the estimation of time-activity curves and kinetic model parameters directly from dynamic SPECT projection data by modeling the spatial and temporal distribution of the radiopharmaceutical throughout the projected field of view.

In previous work we developed computationally efficient methods for fully four-dimensional (4-D) direct estimation of spatiotemporal distributions [1] and their statistical uncertainties [2] from dynamic SPECT projection data, using a spatial segmentation and temporal B-splines. In addition, we studied the bias that results from modeling various orders of temporal continuity and using various time samplings [1]. In the present work, we use the methods developed in $[1,2]$ and Monte Carlo simulations to study the effects of the temporal modeling on the statistical variability of the reconstructed distributions.

\section{FASt COMPUtation of Statistical UnCERTAinty}

Time-varying activity concentrations within volumes of interest encompassing the projected SPECT field of view can be modeled by selecting a set of temporal basis functions capable of representing typical time variations and having desired smoothness properties. Similarly, the spatially nonuniform activity concentration within a particular volume of interest can be modeled by selecting an appropriate set of spatial basis functions. Given a set of temporal basis functions and sets of spatial basis functions for the volumes of interest, coefficients for the resulting spatiotemporal basis functions can be estimated directly from

This work was supported by the National Heart, Lung, and Blood Institute of the US Department of Health and Human Services under grants R01-HL50663 and P01-HL25840 and by the Director, Office of Science, Office of Biological and Environmental Research, Medical Sciences Division of the US Department of Energy under contract DE-AC03-76SF00098. This work was developed in part using the resources at the US Department of Energy National Energy Research Scientific Computing (NERSC) Center. the SPECT projection data [1], along with the covariance matrix for the coefficients [2].

Denoting the projection of the $m^{\text {th }}$ spatial basis function along ray $i$ at angle $j$ by $u_{i j}^{m}$, and the integral of the $n^{\text {th }}$ temporal basis function during the time interval associated with angle $j$ of rotation $k$ by $v_{j k}^{n}$, the projection equations can be expressed as $p_{i j k}=\sum_{m=1}^{M} \sum_{n=1}^{N} a_{m n} u_{i j}^{m} v_{j k}^{n}$, where the $p_{i j k}$ are the modeled projections, the $a_{m n}$ are linear coefficients, and $M, N$ are the numbers of spatial and temporal basis functions, respectively. The coefficients $a_{m n}$ are varied to find the values $\hat{a}_{m n}$ that minimize the sum of squares function $\chi^{2}=$ $\sum_{i=1}^{I} \sum_{j=1}^{J} \sum_{k=1}^{K}\left(p_{i j k}^{*}-p_{i j k}\right)^{2}$, where the $p_{i j k}^{*}$ are the measured projections, $I$ is the number of projection rays per angle, $J$ is the number of angles per rotation, and $K$ is the number of rotations.

The integral of the time-activity curve model for volume of interest $m$, during the time interval associated with angle $j$ of rotation $k$, can be expressed as $\sum_{n=1}^{N} \hat{a}_{m n} v_{j k}^{n}$. Thus, given the covariance matrix for the spatiotemporal basis function coefficients $\hat{a}_{m n}$, it can be shown that the variance of each time integral is

$$
\sigma_{j k m}^{2}=\sum_{n=1}^{N} \sum_{n^{\prime}=1}^{N} v_{j k}^{n} \operatorname{cov}\left(\hat{a}_{m n}, \hat{a}_{m n^{\prime}}\right) v_{j k}^{n^{\prime}} .
$$

Methods for quickly estimating the covariance matrix for the coefficients were presented, benchmarked, and validated in [2].

As a figure of merit related to the global precision of the timeactivity curve model for volume of interest $m$, the following expression yields a squared noise-to-signal ratio (NSR) calculated as the mean (over all of the time intervals) of the expected values of the squared errors between the integrated intervals of the "true" and modeled curves, normalized by the mean square value of the integrated intervals of the "true" curve:

$$
\xi_{m}^{2}=\frac{\sum_{j=1}^{J} \sum_{k=1}^{K} \sigma_{j k m}^{2}}{\sum_{j=1}^{J} \sum_{k=1}^{K}\left[\sum_{n=1}^{N} \hat{a}_{m n} v_{j k}^{n}\right]^{2}} .
$$

Substituting equation (1) into equation (2), the squared NSR, $\xi_{m}^{2}$, can be calculated quickly by rearranging the summations, precomputing the inner products of the temporal basis functions, 


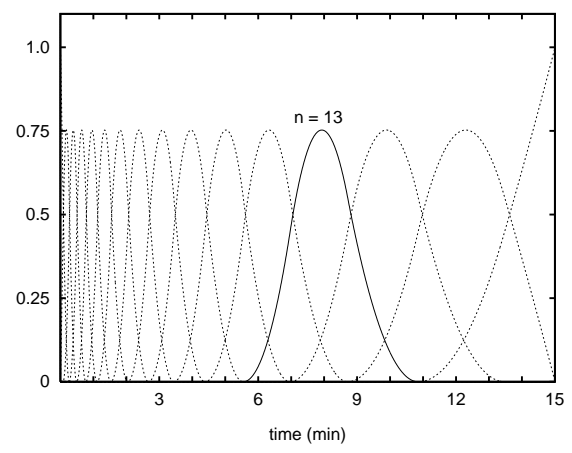

Fig. 1. Quadratic B-spline basis functions with initial segment length of $10 \mathrm{sec}$.

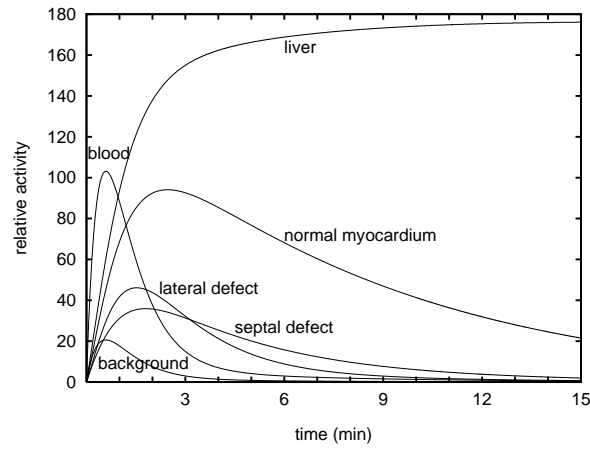

Fig. 2. Simulated teboroxime time-activity curves.

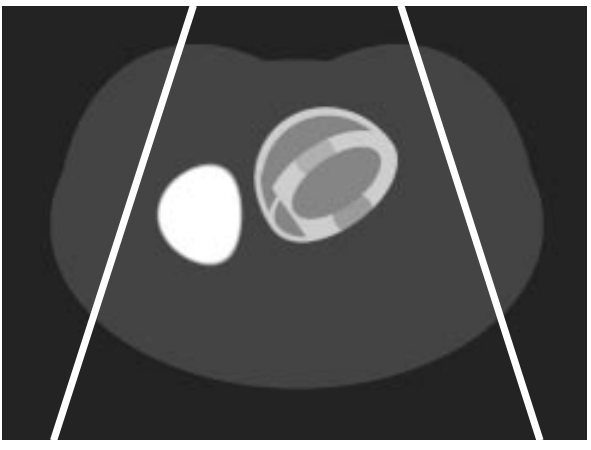

Fig. 3. Cross section through MCAT emission phantom, showing data truncation.
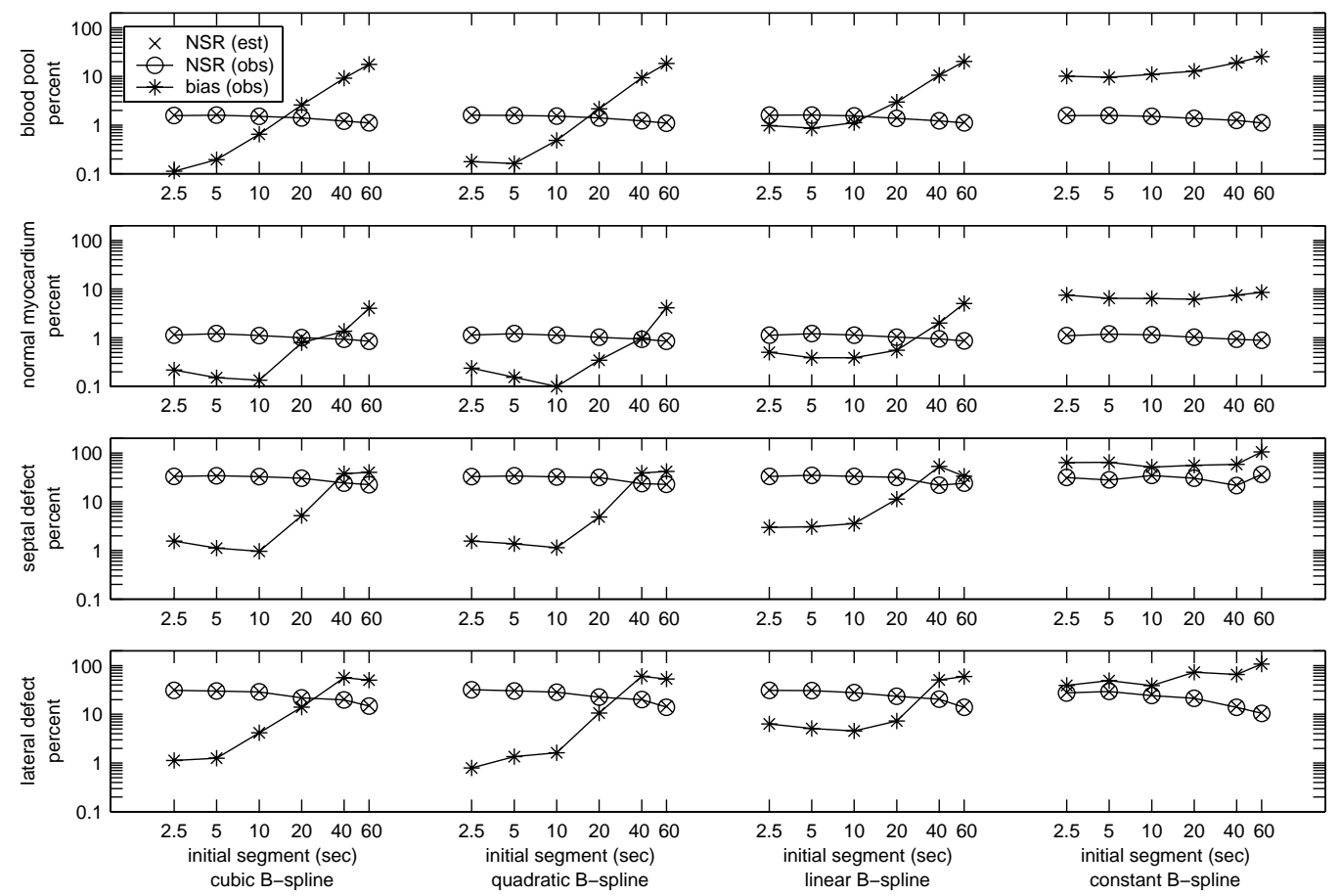

Fig. 4. Results of Monte Carlo simulations. The “*” symbols denote bias values observed for 1000 realizations of noisy projections. For each temporal basis set, bias was calculated by first calculating the mean time-activity curve for the 1000 noisy curves, and then calculating the root mean square (RMS) difference between the mean noisy curve and the simulated curve (Fig. 2). The RMS difference was then normalized by the RMS value for the simulated curve and expressed as a percentage. The "o" symbols denote the observed NSR for each basis set, which was calculated as the mean value of the RMS differences between the 1000 noisy curves and the mean noisy curve, normalized by the RMS value of the mean noisy curve, and expressed as a percentage. The " $\times$ " symbols denote the mean values of the estimated NSR, $\xi_{m}$, calculated using equation (3). These values agree closely with the observed NSR values. $\nu^{n n^{\prime}}=\sum_{j=1}^{J} \sum_{k=1}^{K} v_{j k}^{n} v_{j k}^{n^{\prime}}$, and exploiting the symmetry with respect to the indices $n$ and $n^{\prime}$ :

$$
\xi_{m}^{2}=\frac{\sum_{n=1}^{N} \sum_{n^{\prime}=1}^{N} \operatorname{cov}\left(\hat{a}_{m n}, \hat{a}_{m n^{\prime}}\right) \nu^{n n^{\prime}}}{\sum_{n=1}^{N} \sum_{n^{\prime}=1}^{N} \hat{a}_{m n} \hat{a}_{m n^{\prime}} \nu^{n n^{\prime}}} .
$$

\section{Computer Simulations}

To study the statistical variability that results from modeling various orders of temporal continuity and using various time samplings, 1000 realizations of cone beam projection data having Poisson noise were generated for each of 24 sets of temporal basis functions. Each set of temporal basis functions consisted of $N=16$ splines spanning 15 time segments having geometrically increasing length (Fig. 1). Piecewise cubic, quadratic, linear, or constant B-splines were used with initial time segment lengths of $2.5,5,10,20,40$, or $60 \mathrm{sec}$.

The simulated time-activity curves (Fig. 2) mimicked the kinetics of teboroxime [3]. The simulated $15 \mathrm{~min}$ data acquisition consisted of $I=2048$ cone beam projection rays per angle (64 transverse $\times 32$ axial), $J=120$ angles per revolution, and
$K=15$ revolutions. The amplitude of the blood input function was adjusted so that about 10 million total events were detected.

Simulated spatial distributions were obtained using the Mathematical Cardiac Torso (MCAT) phantom [4]. The emission phantom (Fig. 3) was composed of 128 contiguous $1.75 \mathrm{~mm}$ thick slices and contained the blood pool, three myocardial tissue volumes of interest (normal myocardium, septal defect, and lateral defect), liver, and background tissue. The projection bins were $7 \mathrm{~mm} \times 7 \mathrm{~mm}$ at the detector, and the cone beam geometry resulted in data truncation (Fig. 3). Projections were attenuated using the corresponding MCAT attenuation phantom. Attenuation and geometric point response were modeled using a raydriven projector with line length weighting [5]. Scatter was not modeled. The spatial basis functions were based on the known segmentation of the MCAT phantom. Each volume of interest was modeled to contain spatially uniform activity, which yielded $M=6$ spatial basis functions.

Fig. 4 shows the simulation results for the blood pool and three myocardial tissue volumes. For each temporal basis set and volume of interest, there was close agreement between the sample mean of the estimated NSR, $\xi_{m}$, and the observed NSR, 
which was calculated as described in the caption for Fig. 4. The effect of the polynomial order of the splines on the NSR was relatively small, while the effect of the time sampling was larger. The NSR tended to decrease as the time sampling became more uniform (e.g., for initial time segment lengths of 40 or $60 \mathrm{sec}$ ). However, the decrease in NSR was offset by an increase in bias, which was calculated as described in the caption for Fig. 4. The bias increased because these basis sets had initial samplings that were too long to accurately model the beginning of the acquisition, when the activity concentrations were changing most rapidly (Fig. 2). The bias also tended to increase as the polynomial order of the splines decreased. Overall, the best results were obtained with cubic or quadratic splines having initial time samplings of $10 \mathrm{sec}$ or less. Similar findings were obtained for the liver and background tissue volumes.

\section{Discussion}

The simulation results suggest that there is benefit in modeling higher orders of temporal continuity, when estimating spatiotemporal distributions directly from dynamic SPECT projections. In addition, the accuracy of the time modeling can be increased substantially without unduly increasing the statistical uncertainty, by using relatively rapid initial time sampling.

Future work includes a study of the effects of the B-spline order and initial time sampling on nonlinear weighted least squares estimates of compartmental model kinetic parameters obtained from the time-activity curve models.

\section{ACKNOWLEDGMENT}

The authors thank the University of North Carolina Medical Imaging Research Laboratory for making the MCAT phantom available.

This work was supported by the National Heart, Lung, and Blood Institute of the US Department of Health and Human Services under grants R01-HL50663 and P01-HL25840 and by the Director, Office of Science, Office of Biological and Environmental Research, Medical Sciences Division of the US Department of Energy under contract DE-AC03-76SF00098. This work was developed in part using the resources at the US Department of Energy National Energy Research Scientific Computing (NERSC) Center.

\section{REFERENCES}

[1] B W Reutter, G T Gullberg, and R H Huesman, "Direct least-squares estimation of spatiotemporal distributions from dynamic SPECT projections using a spatial segmentation and temporal B-splines," IEEE Trans Med Imag, vol. 19, no. 5, pp. 434-450, 2000.

[2] B W Reutter, G T Gullberg, and R H Huesman, "Fast computation of statistical uncertainty for spatiotemporal distributions estimated directly from dynamic cone beam SPECT projections," Tech. Rep. LBNL-47761, Lawrence Berkeley National Laboratory, 2001 (submitted to the Sixth International Meeting on Fully 3-D Image Reconstruction in Radiology and Nuclear Medicine, and available at http://cfi.lbl.gov/data_analysis/pub_list).

[3] R K Narra, T Feld, and A D Nunn, "Absorbed radiation dose to humans from technetium-99m-teboroxime," J Nucl Med, vol. 33, no. 1, pp. 88-93, 1992.

[4] B M W Tsui, J A Terry, and G T Gullberg, "Evaluation of cardiac conebeam single photon emission computed tomography using observer performance experiments and receiver operating characteristic analysis," Invest Radiol, vol. 28, no. 12, pp. 1101-1112, 1993.

[5] G L Zeng, G T Gullberg, B M W Tsui, and J A Terry, "Three-dimensional iterative reconstruction algorithms with attenuation and geometric point response correction," IEEE Trans Nucl Sci, vol. 38, no. 2, pp. 693-702, 1991.

\section{DISCLAIMER}

This document was prepared as an account of work sponsored by the United States Government. While this document is believed to contain correct information, neither the United States Government nor any agency thereof, nor The Regents of the University of California, nor any of their employees, makes any warranty, express or implied, or assumes any legal responsibility for the accuracy, completeness, or usefulness of any information, apparatus, product, or process disclosed, or represents that its use would not infringe privately owned rights. Reference herein to any specific commercial product, process, or service by its trade name, trademark, manufacturer, or otherwise, does not necessarily constitute or imply its endorsement, recommendation, or favoring by the United States Government or any agency thereof, or The Regents of the University of California. The views and opinions of authors expressed herein do not necessarily state or reflect those of the United States Government or any agency thereof, or The Regents of the University of California.

Ernest Orlando Lawrence Berkeley National Laboratory is an equal opportunity employer. 